\title{
Phase correction of VLBI with water vapour radiometry
}

\author{
Alan L. Roy* \\ Max-Planck-Institut für Radioastronomie, Auf dem Hügel 69, 53121 Bonn, Germany \\ E-mail: aroy@mpifr-bonn.mpg.de \\ Helge Rottmann \\ Max-Planck-Institut für Radioastronomie, Auf dem Hügel 69, 53121 Bonn, Germany \\ E-mail: rottmann@mpifr-bonn.mpg.de

\section{Ute Teuber} \\ Max-Planck-Institut für Radioastronomie, Auf dem Hügel 69, 53121 Bonn, Germany \\ E-mail: uteuber@mpifr-bonn.mpg.de

\section{Reinhard Keller} \\ Max-Planck-Institut für Radioastronomie, Auf dem Hügel 69, 53121 Bonn, Germany \\ E-mail: rkeller@mpifr-bonn.mpg.de
}

\begin{abstract}
We demonstrate phase correction of 3-mm VLBI observations using the scanning 18-GHz to 26$\mathrm{GHz}$ water vapour radiometer (WVR) at Effelsberg and we demonstrate an absolute accuracy of $15-\mathrm{mm}$ in zenith path delay by comparing with GPS and radiosondes. This accuracy should provide significant improvement in astrometric phase-referencing observations. It is not good enough for geodetic VLBI to replace the tropospheric delay estimation but could be used to remove short-term path-length fluctuations and so improve the geodetic observables. We discuss lessons learned and opportunities for further improvement.
\end{abstract}

The 8th European VLBI Network Symposium

September 26-29, 2006

Toruń, Poland

${ }^{*}$ Speaker. 


\section{Introduction}

In March 2004, the Effelsberg radio telescope was equipped with a 22-GHz water vapour radiometer (WVR) for phase correction of tropospheric phase fluctuations during VLBI experiments at frequencies up to $86 \mathrm{GHz}$. The radiometer also measures tropospheric path delay for astrometric and geodetic VLBI experiments. It has been used during routine VLBI experiments at $86 \mathrm{GHz}$ for validation tests and software is being developed to provide the data to observers on a regular basis.

VLBI at $86 \mathrm{GHz}$ yields resolution down to $60 \mu$ as with global baselines. Experiments up to $230 \mathrm{GHz}$ have produced fringes with a record smallest spacing of $30 \mu \mathrm{as}$, on the $6.4 \mathrm{G} \lambda$ transatlantic baseline between Pico Veleta and the HHT (Krichbaum et al. 2004), which is comparable to the $27 \mu$ as diameter predicted by Falcke, Melia \& Agol (2000) for the silhouette of the event horizon in Sgr $\mathrm{A}^{*}$. However, the signal-to-noise ratios of the $230-\mathrm{GHz}$ detections were only 7.3 on on $3 \mathrm{C} 454.3$ and 6.4 on $0716+714$ due to a combination of decoherence due to atmospheric phase fluctuations during the 7-min integration, relatively high system noise, and atmospheric opacity. Sensitivity could be improved by a factor of two to four by correcting for the coherence loss due to atmospheric phase noise using water vapour radiometry. Water vapour radiometry monitors the strength of emission from atmospheric water vapour with high precision and infers a timedependent correction to the VLBI phase, which then improves the temporal coherence.

\section{The water vapour radiometer at Effelsberg (WAVE)}

We have built a scanning $18-\mathrm{GHz}$ to $26-\mathrm{GHz}$ water vapour radiometer and installed it on the focus cabin roof at Effelsberg viewing along the optical axis. It is based on a prototype by Alan Rogers that was demonstrated at BIMA and during 3-mm VLBI on the baseline between BIMA and Kitt Peak (Tahmoush \& Rogers 2000). The radiometer is uncooled to reduce cost, is scanning to reduce the parts count and hence cost, and to provide better stability since offsets and drifts affect all channels and so are common-mode noise sources, which are rejected to some extent when differencing on-line and off-line channels. We paid particular attention to RF shielding, weather proofing, temperature stabilization using a Peltier element to maintain the $\mathrm{RF}$ electronics near $30^{\circ} \mathrm{C}$ and we added a noise diode for noise-adding radiometry to monitor the system gain. Monitoring and control takes place over a TCP/IP connection on optical fibre, so the system requires only power and a network connection to operate. The data are logged into a MySQL database from where they can be examined and downloaded through a web interface.

Photographs, schematics, measured performance and correction of atmospheric opacity have been shown by Roy et al. (2004). Here, we demonstrate the correction of tropospheric phase fluctuations during an 86-GHz VLBI experiment and we validate the absolute calibration for astrometric and geodetic application by comparing to GPS and radiosonde measurements.

\section{Demonstration of phase correction}

A typical water line spectrum measured by the radiometer is shown in Fig. 1a, as presented by the web interface to the WVR database. The line is centred on $22.2 \mathrm{GHz}$ and is pressure-broadened to $\sim 3 \mathrm{GHz}$ FWHM. The line appears asymmetric as it sits on the frequency-squared wing of 

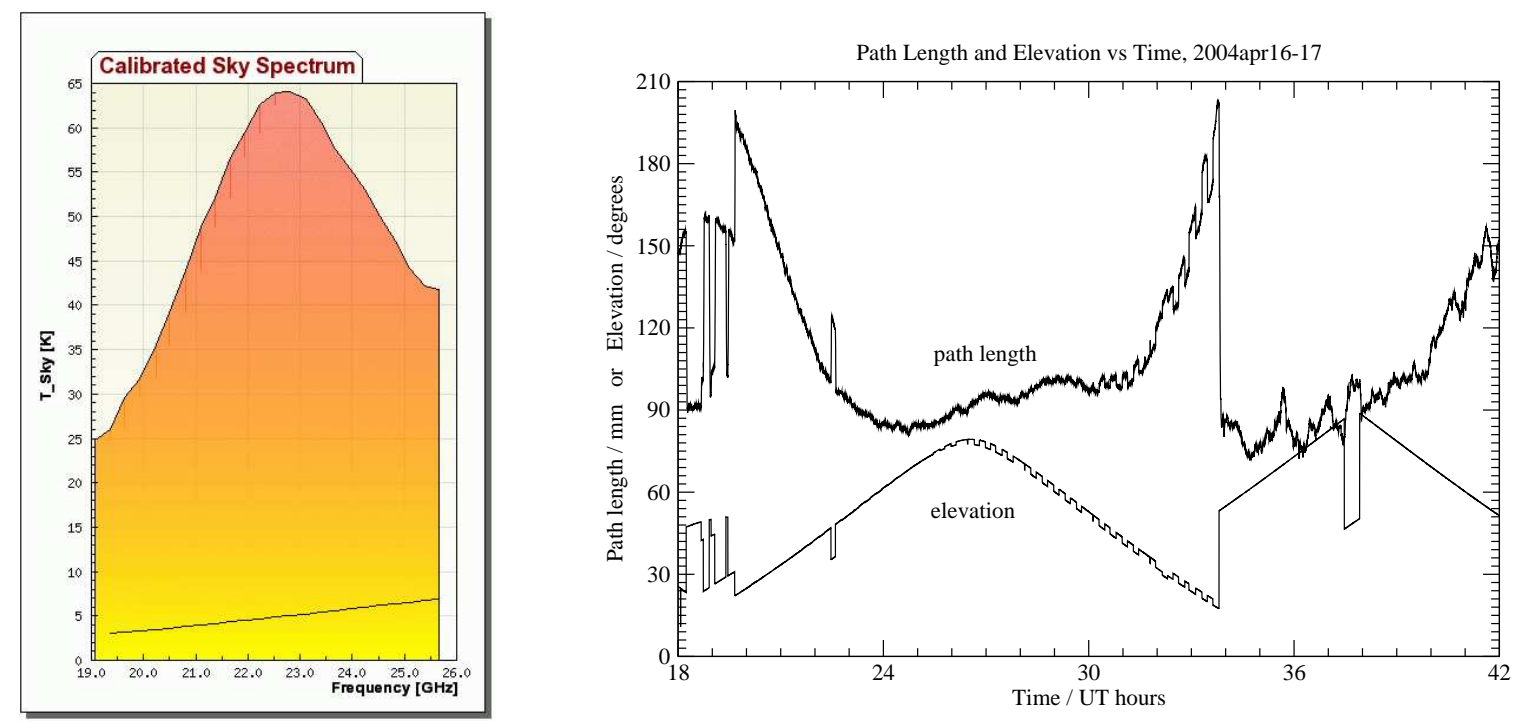

Figure 1: a) (left): An example 22-GHz water line spectrum measured with the water vapour radiometer at Effelsberg on $20 \mathrm{Jul} 2006$ 11:56 UT at an elevation of 54 ${ }^{\circ}$ under cloud-free sky. The line curving upwards at the bottom is one of three components (the frequency-squared baseline) that was fit to the spectrum. b) (right): An example time series of tropospheric line-of-sight path length derived from the line strength measurements made with the WVR at Effelsberg on 16/17 Apr 2004 during an 86-GHz VLBI experiment.

the oxygen line at $60 \mathrm{GHz}$. Such a spectrum is measured each $6 \mathrm{~s}$. To each spectrum, we fit a three-component model consisting of a frequency-squared baseline underneath the water line plus a constant offset and a van Vleck-Weisskopf profile to the line to estimate the strength of the line above the baseline. From the line strength, we infer the path length using the theoretical relationship between refractive index and emissivity of water vapour derived by Tahmoush \& Rogers (2000).

A typical time series of tropospheric path length inferred from the WVR is presented in Fig. 1b. This shows a 24-hour period during the 86-GHz VLBI experiment on 16/17 Apr 2004. The path length varies with elevation as the airmass changes and the short-term path-length fluctuations grow throughout the period due to a change in the weather.

A 7-min piece of the time series is shown in Fig. 2a along with the measured VLBI phase on the Effelsberg - Pico Veleta baseline. The inferred path length and the observed VLBI phase correlate strongly. Correcting the VLBI phase with the WVR corrections reduced the path rms from $1.0 \mathrm{~mm}$ rms to $0.47 \mathrm{~mm} \mathrm{rms}$ and raised the coherence from 0.45 to 0.86 for a $240 \mathrm{~s}$ timescale. Phase correction data from the Pico Veleta 230-GHz continuum radiometer described by Bremer (2002) were applied, further reducing the rms path fluctuations to $0.34 \mathrm{~mm}$ and raising the coherence to 0.9 . In total, the coherent signal-to-noise ratio improved by a factor of 2.1 .

Another scan during cloud is shown in Fig. 2b. The phase corrections varied dramatically and no phase correlation could be seen. We are now improving the cloud separation. Phase correction in the presence of cloud has been reported by Bremer (2006) at Plateau de Bure.

The fraction of scans for which the WVR corrections improved the coherence is summarized in Fig. 2c. It shows the coherence after correction divided by the coherence before correction. If the correction makes an improvement, the ratio is greater than unity. Most scans lie between 1 and 

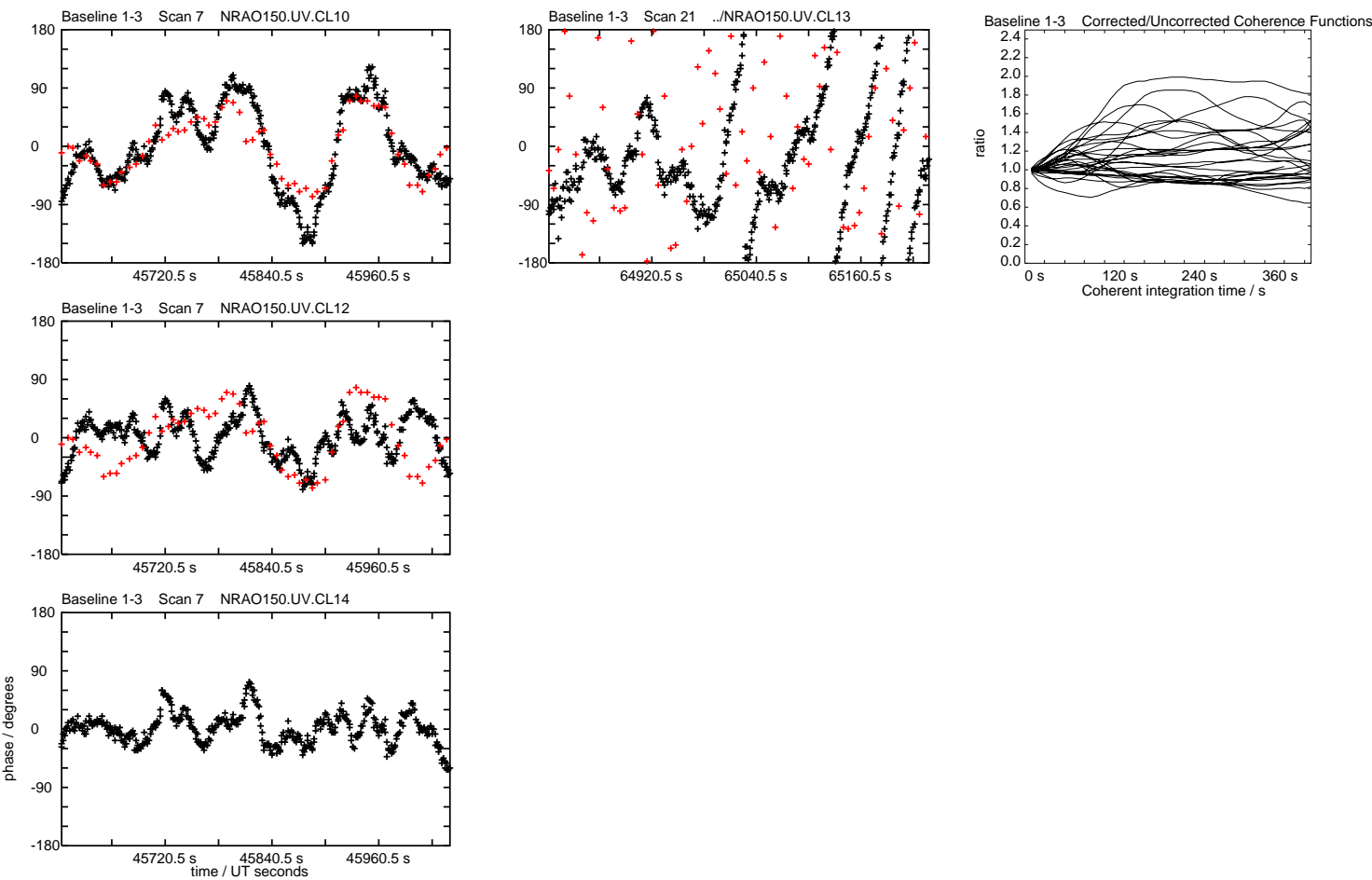

Figure 2: a) (left column): Phase correction of a 7-min long 86-GHz VLBI scan on NRAO 150 on the baseline Pico Veleta - Effelsberg on 17 Apr 2004 at 12.7 h UT. Left top: the VLBI phase time series in black and the WVR-derived phase correction in red show good correlation. Left middle: the VLBI phase time series after applying the Effelsberg WVR correction shows reduced phase fluctuations. Left bottom: phase corrections at Pico Veleta based on the measured continuum brightness at $230 \mathrm{GHz}$ made in parallel with the 86-GHz VLBI observations are applied to the middle plot, further reducing the phase fluctuations. The rms path fluctuations reduced from $1.0 \mathrm{~mm}$ to $0.34 \mathrm{~mm}$ and the coherence rose from 0.45 to 0.9 over a $240-\mathrm{s}$ time-scale. b) (centre column): Phase correction of another 86-GHz VLBI scan on NRAO 150 on the Pico Veleta - Effelsberg baseline on 17 Apr 2004 at $18.0 \mathrm{~h}$ UT this time affected by cloud emission that was not separated and so contaminated the path length measurement. c) (right column): The ratio of coherence after and before phase correction is plotted vs coherent integration time for all scans on NRAO 150 on 17 Apr 2004, excluding those affected by cloud based on the high rms in the time series. A ratio $>1$ indicates improvement. Most scans were improved by the phase correction.

2 , with the worst case being 0.75 . Scans affected by cloud were removed for this summary.

Corrections applied to four subsequent 86-GHz VLBI experiments yielded no further cases of improved coherence; in fact, in these four experiments, applying WVR corrections even increased the rms phase noise. The cause is under investigation; perhaps the atmospheric stability during those experiments was already very good and the WVR noise raised the rms. This is plausible since the WVR corrections were often seen to be stable and the VLBI phase wandered by rather more suggesting that the phase noise was dominated by the contribution from the other end of the baseline. Other observatories have also seen periods when the phase corrections from WVRs fail to reduce the tropospheric phase noise at the VLA (Chandler et al. 2004), Plateau de Bure (Bremer 2006), and OVRO (Woody, Carpenter \& Scoville 2000) though not as often as in our tests. 


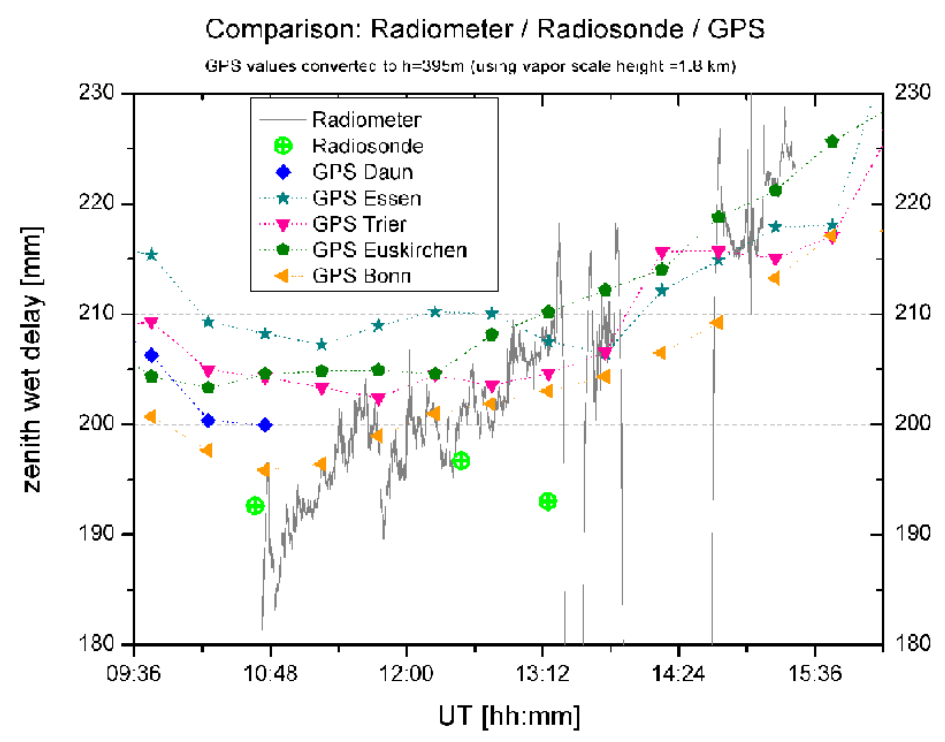

Figure 3: Time series of zenith wet delay measured by the Effelsberg WVR, GPS receivers between 18-km and 120-km distance from the Effeslberg telescope, and radiosondes launched $400 \mathrm{~m}$ ENE of the telescope. The three techniques agree typically within $15 \mathrm{~mm}$. The third radiosonde profile (at 13:12 UT) was incomplete due to interrupted data reception. Hence, this one measurement has larger uncertainty.

\section{Validation of absolute calibration}

Astrometric and geodetic applications of water vapour radiometry require good absolute calibration; low thermal noise is less important. To check the absolute calibration, we carried out an intercomparison of WVR, five GPS stations closest to Effelsberg out to $120 \mathrm{~km}$ and three radiosonde launches on 27 Jul 2005. The GPS data were analyzed at GeoForschungsZentrum, Potsdam and the radiosonde data were analyzed by C. Simmer at the Dept. of Meteorology, Bonn University to derive zenith tropospheric path delays. We found agreement within $15 \mathrm{~mm}$ between the WVR, all GPS receivers and three radiosonde launches (Fig. 3).

\section{Lessons learned}

We came to appreciate the critical importance of good temperature stabilization for maintaining absolute calibration and maintaining the $3 \times 10^{-4}$ short-term gain stability required for phase correction. We had initially $0.7^{\circ} \mathrm{C}$ variation in the internal temperature over a 24 -h period, however superimposed on this was a temperature oscillation with a 3-min period and peak-to-peak amplitude of $20 \mathrm{mK}$ due to the time lag for heat flow from the Peltier element to the temperature sensor located beside the LNA. The temperature oscillation caused the detector output power to vary by $250 \mathrm{mK}$ peak-to-peak which was enough to degrade the phase correction. We stopped the oscillation by weakening the thermal contact between the Peltier element and the RF components by reducing the speed of a fan and removing copper straps but the internal temperature then followed the external temperature more closely, producing $6^{\circ} \mathrm{C}$ or more temperature changes over periods of days. Much tighter temperature regulation has been demonstrated by, for example, Tanner (1998). 
The frequency spanned by the radiometer might be too narrow to allow separation of cloud emission with the precision required to allow phase correction to continue during periods of heavy cloud. A channel on the continuum at $50 \mathrm{GHz}$ would help this a lot (Crewell 2006). However, phase correction in the presence of clouds with a narrower frequency span than ours has been demonstrated by Bremer (2006) at Plateau de Bure, thus proving the principle.

The spillover contribution is unexpectedly large $(8 \mathrm{~K})$ since the feedhorn over-illuminates the dish at low frequencies. A new horn with larger edge taper will alleviate this source of noise.

The noise diode proved somewhat unstable, and the system gain variations could be better removed using the temperature measured near the LNA along with the temperature coefficient of the amplifier. Methods to improve the noise diode stability have been described by Tanner (1998).

\section{Conclusion}

The Effelsberg water vapour radiometer corrections have been demonstrated to improve phase coherence during one high-frequency VLBI experiment, though not in four subsequent experiments. The tropospheric delay measurements agreed with $15 \mathrm{~mm}$ accuracy when compared to GPS and radiosondes.

\section{Acknowledgments}

We thank Clemens Simmer (Dept. of Meteorology, Bonn University) for carrying out the radiosonde launches at Effelsberg and for analyzing the data to derive zenith path delays. We had a lot of fun. We also thank Galina Dick and her colleagues at GeoForschungsZentrum, Potsdam for analyzing the GPS data to derive zenith path delays for comparison with those from the radiometer. We also thank Ivan Agudo for providing us with the 86-GHz VLBI data on NRAO 150 with which we tested the WVR phase corrections.

\section{References}

[1] M. Bremer 2002, Atmospheric Phase Correction for Connected-Element Interferometry and for VLBI in Astronomical Site Evaluation in the Visible and Radio Range. ASP Conference Proceedings, Vol. 266, eds J. Vernin, Z. Benkhaldoun, and C. Muñoz-Tuñón. (San Francisco: Astronomical Society of the Pacific) p238

[2] M. Bremer 2006, in Proc. RadioNet Workshop on Measurement of Atmospheric Water Vapour: Theory, Techniques, Astronomical and Geodetic Applications, Wettzell / Hoellenstein, October 9-11 2006, http://www.wettzell.ifag.de/veranstaltungen/wvr/workshop2006/wvr2006.html

[3] C. J. Chandler, W. F. Brisken, B. J. Butler, R. H. Hayward \& B. E. Willoughby 2004, EVLA Memo 73

[4] S. Crewell 2006, in Proc. RadioNet Workshop on Measurement of Atmospheric Water Vapour: Theory, Techniques, Astronomical and Geodetic Applications, Wettzell / Hoellenstein, October 9-11 2006, http://www.wettzell.ifag.de/veranstaltungen/wvr/workshop2006/wvr2006.html

[5] H. Falcke, F. Melia, \& E. Agol 2000, ApJ, 528, L13

[6] T. P. Krichbaum, D. A. Graham, W. Alef et al. 2004, Towards the Event Horizon - The Vicinity of AGN at Micro-Arcsecond Resolution in Proc. 7th EVN Symposium, Toledo (Spain), October 12-15 2004, eds R. Bachiller, F. Colomer, J.-F. Desmurs \& P. de Vicente, (OAN: Spain), p15 
[7] A. L. Roy, U. Teuber, \& R. Keller 2004, The Water Vapour Radiometer at Effelsberg in Proc. 7th EVN Symposium on New Developments in VLBI Science and Technology, Toledo (Spain), October 12-15 2004, eds R. Bachiller, F. Colomer, J.-F. Desmurs \& P. de Vicente, (OAN: Spain), p265

[8] D. A. Tahmoush \& A. E. E. Rogers 2000, Radio Sci, 35, 1241

[9] A. B. Tanner 1998, Radio Sci, 33, 449

[10] D. Woody, J. Carpenter \& N. Scoville 2000, Phase Correction at OVRO Using 22 GHz Water Line Monitors in Imaging at Radio through Submillimeter Wavelengths ASP Conference Proceedings, Vol. 217, eds J. G. Mangum \& S. J. E. Radford, (San Francisco: ASP) p317 